\title{
Global Observations from PHOBOS
}

\author{
Mark D. Baker for the PHOBOS Collaboration
}

B.B.Back ${ }^{1}$, M.D.Baker ${ }^{2}$, D.S.Barton ${ }^{2}$, R.R.Betts ${ }^{6}$, M.Ballintijn $^{4}$, A.A.Bickley ${ }^{7}$, R.Bindel $^{7}$, A.Budzanowski $^{3}$, W.Busza $^{4}$, A.Carroll ${ }^{2}$, M.P.Decowski ${ }^{4}$, E.García ${ }^{6}$, N.George $^{2}$, K.Gulbrandsen $^{4}$, S.Gushue $^{2}$, C.Halliwell $^{6}$, J.Hamblen $^{8}$, G.A.Heintzelman ${ }^{2}$, C.Henderson ${ }^{4}$, D.J.Hofman ${ }^{6}$, R.S.Hollis ${ }^{6}$, R.Hołyński ${ }^{3}$, B.Holzman ${ }^{2}$, A.Iordanova $^{6}$, E.Johnson ${ }^{8}$, J.L.Kane ${ }^{4}$, J.Katzy ${ }^{4,6}$, N.Khan ${ }^{8}$, W.Kucewicz ${ }^{6}$, P.Kulinich ${ }^{4}$, C.M.Kuo ${ }^{5}$, W.T.Lin ${ }^{5}$, S.Manly ${ }^{8}$, D.McLeod ${ }^{6}$, J.Michałowski ${ }^{3}$, A.C.Mignerey ${ }^{7}$, R.Nouicer ${ }^{6}$, A.Olszewski ${ }^{3}$, R.Pak ${ }^{2}$, I.C.Park ${ }^{8}$, H.Pernegger ${ }^{4}$, C.Reed ${ }^{4}$, L.P.Remsberg ${ }^{2}$, M.Reuter ${ }^{6}$, C.Roland ${ }^{4}$, G.Roland ${ }^{4}$, L.Rosenberg $^{4}$, J.Sagerer ${ }^{6}$, P.Sarin ${ }^{4}$, P.Sawicki ${ }^{3}$, W.Skulski ${ }^{8}$, S.G.Steadman ${ }^{4}$, P.Steinberg ${ }^{2, b}$, G.S.F.Stephans ${ }^{4}$, M.Stodulski ${ }^{3}$, A.Sukhanov ${ }^{2}$, J.-L.Tang ${ }^{5, a}$, R.Teng ${ }^{8}$, A.Trzupek ${ }^{3}$, C.Vale ${ }^{4}$, G.J.van Nieuwenhuizen ${ }^{4}$, R.Verdier ${ }^{4}$, B.Wadsworth ${ }^{4}$, F.L.H.Wolfs ${ }^{8}$, B.Wosiek ${ }^{3}$, K.Woźniak ${ }^{3}$, A.H.Wuosmaa ${ }^{1, c}$, B.Wysłouch $^{4}$

1 Argonne National Laboratory, Argonne, IL 60439-4843, USA

2 Brookhaven National Laboratory, Upton, NY 11973-5000, USA

${ }^{3}$ Institute of Nuclear Physics, Kraków, Poland

${ }^{4}$ Massachusetts Institute of Technology, Cambridge, MA 02139-4307, USA

${ }^{5}$ National Central University, Chung-Li, Taiwan

${ }^{6}$ University of Illinois at Chicago, Chicago, IL 60607-7059, USA

7 University of Maryland, College Park, MD 20742, USA

${ }^{8}$ University of Rochester, Rochester, NY 14627, USA

${ }^{a}$ Current address: National Chung-Cheng University, Chia-Yi, Taiwan

${ }^{b}$ Current address: University of Cape Town, South Africa

${ }^{c}$ Current address: Western Michigan University, Kalamazoo, MI 49008, USA

Particle production in $\mathrm{Au}+\mathrm{Au}$ collisions has been measured in the PHOBOS experiment at RHIC for a range of collision energies. Three empirical observations have emerged from this dataset which require theoretical examination. First, there is clear evidence of limiting fragmentation. Namely, particle production in central $\mathrm{Au}+\mathrm{Au}$ collisions, when expressed as $d N / d \eta^{\prime}\left(\eta^{\prime} \equiv \eta-y_{\text {beam }}\right)$, becomes energy independent at high energy for a broad region of $\eta^{\prime}$ around $\eta^{\prime}=0$. This energy-independent region grows with energy, allowing only a limited region (if any) of longitudinal boost-invariance. Second, there is a striking similarity between particle production in $\mathrm{e}^{+} \mathrm{e}^{-}$and $\mathrm{Au}+\mathrm{Au}$ collisions (scaled by the number of participating nucleon pairs). Both the total number of produced particles and the longitudinal distribution of produced particles are approximately the same in $\mathrm{e}^{+} \mathrm{e}^{-}$and in scaled $\mathrm{Au}+\mathrm{Au}$. This observation was not predicted and has not been explained. Finally, particle production has been found to scale approximately with the number of participating nucleon pairs for $\left\langle N_{\text {part }}\right\rangle>65$. This scaling occurs both for the total multiplicity and for high $p_{T}$ particles $\left(3<p_{T}<4.5 \mathrm{GeV} / \mathrm{c}\right)$. 


\section{INTRODUCTION}

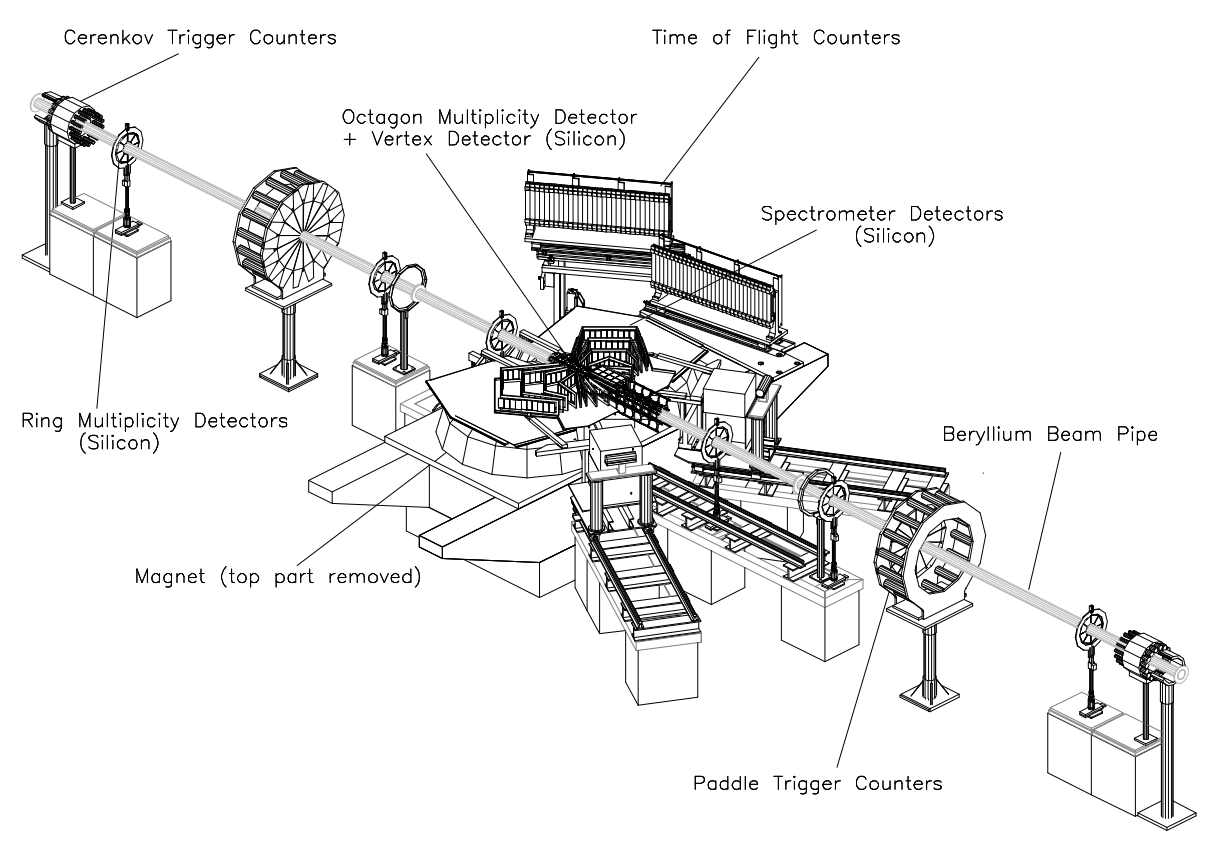

Figure 1. Schematic diagram of the detector setup for the 2001 running period.

The data described in this paper were taken during the Year 2000 and Year 2001 runs of the Relativistic Heavy Ion Collider at Brookhaven National Laboratory. The PHOBOS apparatus [1], shown schematically in Figure 1, was used to take data at three energies: $\sqrt{s_{N N}}=19.6,130$, and $200 \mathrm{GeV}$. A partial test detector was also used to take data at $\sqrt{s_{N N}}=56 \mathrm{GeV}$ [2].

The full PHOBOS apparatus comprises several subdetectors. Silicon detectors are employed for vertex finding, particle tracking and multiplicity measurements. This set of detectors has nearly full azimuthal coverage over a large pseudorapidity range $|\eta|<5.4$. The detector setup also includes two sets of 16 scintillator counters ("paddle counters") located at $\pm 3.21 \mathrm{~m}$ relative to the nominal interaction point along the beam $(z)$ axis. These counters cover pseudorapidity in the range $3<|\eta|<4.5$ and served as the primary event trigger. The collision centrality is characterized by the average number of nucleon participants $\left\langle N_{\text {part }}\right\rangle$, determined as described in Refs. [3, [4]. These references also tabulate the values and systematic errors of $\left\langle N_{\text {part }}\right\rangle$ for each centrality bin.

\section{SURVEY OF BASIC MEASUREMENTS}

A key goal in the design of the PHOBOS experiment was to perform a broad and systematic survey of hadronic particle production in heavy ion collisions. While this survey is still incomplete in terms of energies and species explored, significant progress has been made toward this goal. This section summarizes the basic measurements available so far: multiplicity, particle spectra and azimuthal asymmetry of particle production. 
The pseudorapidity densities $\left(d N_{c h} / d \eta\right)$ and particle yields $\left(d^{2} N / d y d p_{T}\right)$ given here refer to primary produced particles and do not include feed-down products from weak decays of neutral strange particles. Corrections were made for residual effects from secondary interactions and weak decay feed-down as well as for particles which were absorbed or produced in the material surrounding the collision (primarily the Beryllium beampipe and the magnet steel).

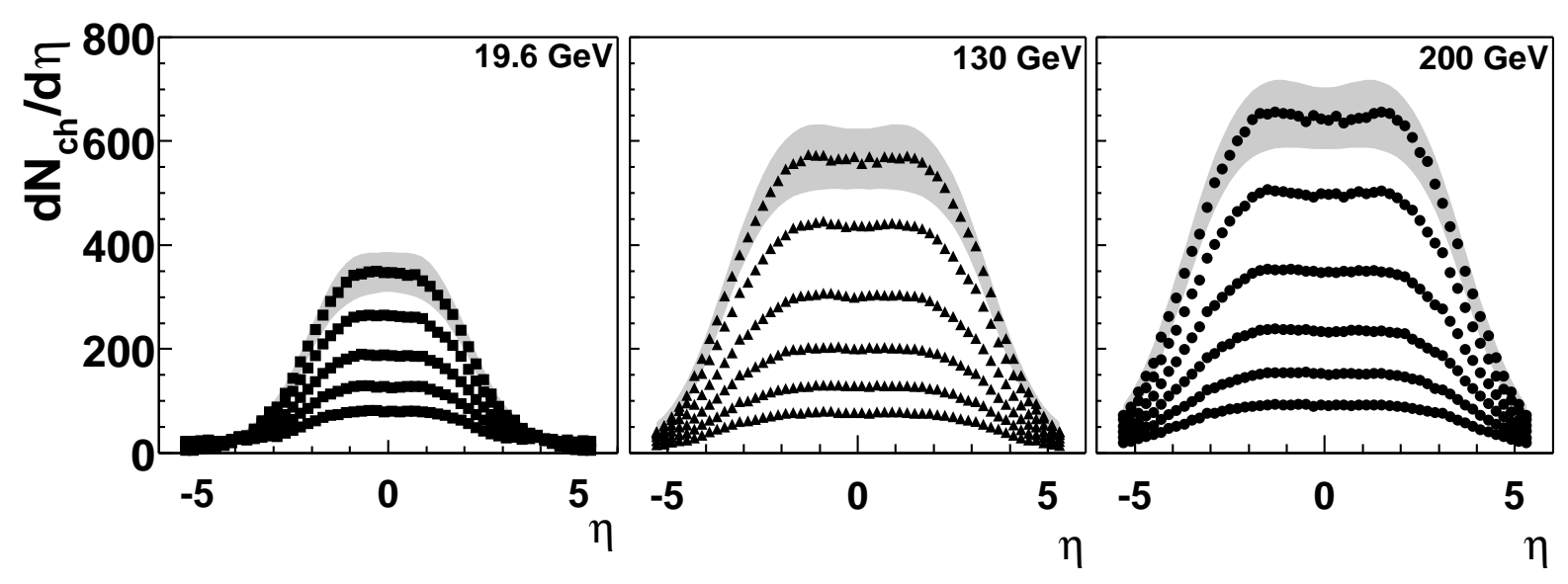

Figure 2. Charged particle pseudorapidity distribution, $d N_{c h} / d \eta$, measured for $\mathrm{Au}+\mathrm{Au}$ at $\sqrt{s_{N N}}=19.6,130$, and $200 \mathrm{GeV}$ for the centrality bins: 0-6\%, 6-15\%, 15-25\%, 25$35 \%, 35-45 \%, 45-55 \%$. The most peripheral bin is excluded for the lowest energy. The statistical error is negligible. The typical systematic error is shown as a 90\% C.L. band for selected centrality bins.

The unique features of the PHOBOS experiment relevant for the charged particle multiplicity measurements are the large acceptance of the detector and the $12 \mathrm{~m}$ long, $1 \mathrm{~mm}$ thick, Beryllium beampipe which limits the absorption of low momentum particles and the production of secondaries. Figure 2 shows the charged particle pseudorapidity distributions $\left(d N_{c h} / d \eta\right)$ measured at $\sqrt{s_{N N}}=19.6,130$, and $200 \mathrm{GeV}$ for a variety of centrality bins for pseudorapidity in the range $-5.4<\eta<5.4$ [4]. Due to the large coverage in $\eta, d N_{c h} / d \eta$ is measured over almost the full range, except for a small missing fraction at very high $|\eta|$. For central events, this missing fraction is estimated to be less than $2 \%$. The multiplicity results are discussed further in Sections 3 and 4 .

Figure 3 shows the results of elliptic flow as a function of pseudorapidity from PHOBOS for 130 and $200 \mathrm{GeV}$. A unique feature of these results is the broad pseudorapidity reach covering almost 11 units of pseudorapidity and showing that there is no broad boostinvariant region near mid-rapidity. The error bars represent $1 \sigma$ statistical errors and the boxes represent $90 \%$ confidence level systematic errors for the $200 \mathrm{GeV}$ data points. These results were measured using a hit-based analysis [5]. A new, track-based, analysis is also available both as a cross-check and to provide $v_{2}$ as a function of particle $p_{T}$ [6].

Figure 4 shows the unidentified charged particle yield measured by PHOBOS for a range 


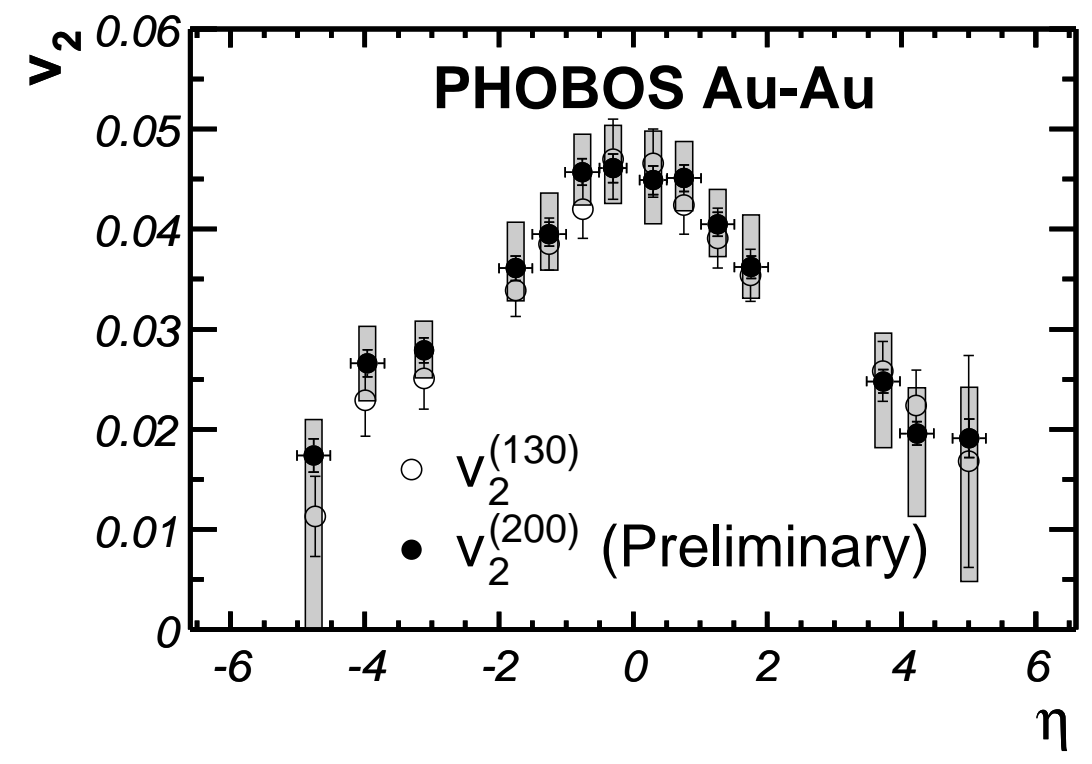

Figure 3. Elliptic flow as a function of pseudorapidity for Au-Au collisions at $\sqrt{s_{N N}}=$ 130 and $200 \mathrm{GeV}$.

of centralities, with rapidity, $y_{\pi}$, defined using the pion mass. These data cover rapidity in the range $0.2<y_{\pi}<1.4$ and transverse momenta in the range $0.2<p_{T}<5.0 \mathrm{GeV} / \mathrm{c}$, corresponding to six decades of change in the magnitude of the yield. Figure 5 shows the invariant yields at very low $p_{T}$ measured by PHOBOS [7]. These particles, which make it through the beampipe and then range out in the active Silicon detectors in the field free region of the spectrometer, are mass-identified but not charge-identified. The yields are shown for $\left(\pi^{ \pm}\right),\left(K^{ \pm}\right)$and $(p+\bar{p})$ measured at mid-rapidity $(-0.1<y<0.4)$ in the transverse momentum ranges from 30 to $50 \mathrm{MeV} / \mathrm{c}$ for charged pions, 90 to $130 \mathrm{MeV} / \mathrm{c}$ for kaons and 140 to $210 \mathrm{MeV} / \mathrm{c}$ for protons and antiprotons for the $15 \%$ most central $\mathrm{Au}+\mathrm{Au}$ collisions at $\sqrt{s_{N N}}=200 \mathrm{GeV}$.

The unique features of PHOBOS relevant for the momentum-measured particles are the close proximity of the detector to the interaction region $(10-80 \mathrm{~cm})$, the precise vertex determination $(0.1-0.3 \mathrm{~mm})$, good segmentation $(0.4-1 \mathrm{~mm})$ of the silicon detectors in the bend direction and, again, the small amount of material between the interaction and the first layers of silicon. These features allow the measurement of particles with good momentum resolution over a broad range of transverse momenta from $0.03-5.0 \mathrm{GeV} / \mathrm{c}$ and beyond (when statistics allow), as well as providing the ability to reject most secondaries and decay products.

The broad $p_{T}$ range, particularly the coverage to very low $p_{T}$, will be important for constraining the role of dynamical processes such as rescattering and radial expansion in these collisions. The charged particle multiplicity (and elliptic flow) measurement spans roughly 11 units of pseudorapidity, $2 \pi$ in azimuth, and a factor of 10 in beam energy, in a single experiment, providing strong constraints on any description of the initial state and subsequent dynamics. From this broad dataset, three empirical scaling rules have 


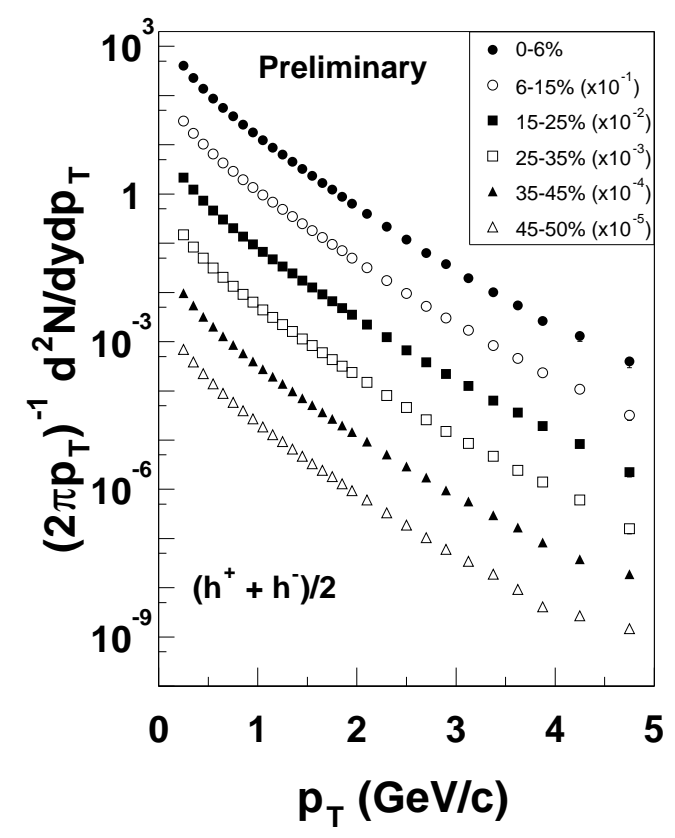

Figure 4. Charged particle invariant yields in $\mathrm{Au}+\mathrm{Au}$ collisions for a variety of centrality ranges. Non-central data have been scaled by arbitrary factors labeled on the plot for clarity.

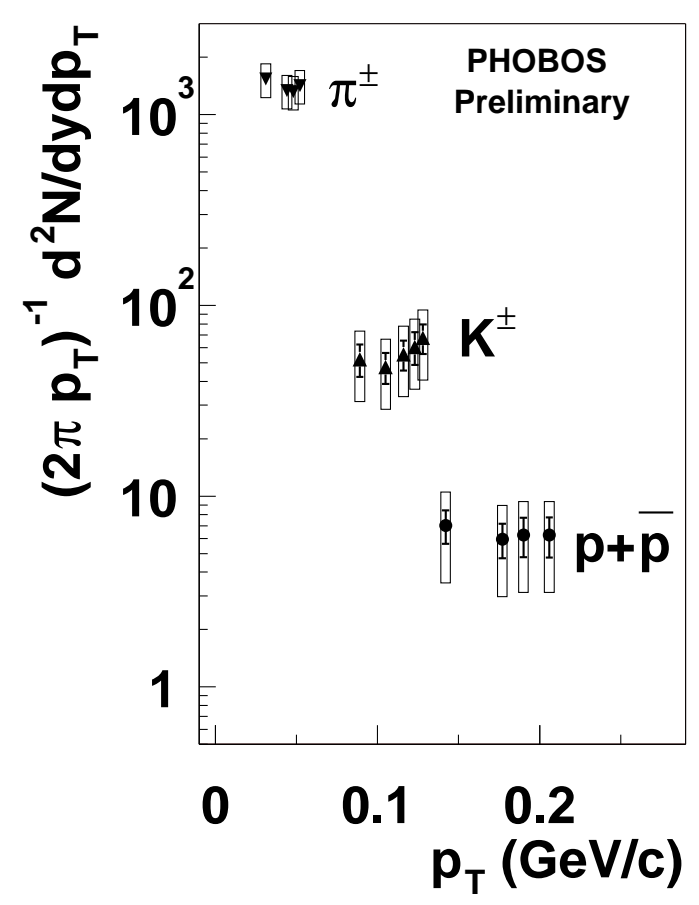

Figure 5. Identified particle invariant yields as a function of $p_{T}$ for central $(0-$ $15 \%) \mathrm{Au}+\mathrm{Au}$ collisions. The boxes show systematic uncertainties.

emerged. Sections 35 describe each of them in turn. These rules, at the very least, provide a compact description of the data which must be respected by any model. At best, these empirical scaling rules may point the way to a more accurate description of the dynamics of these collisions.

\section{THE LIMITING CURVE FOR PARTICLE PRODUCTION}

The pseudorapidity distributions were shown in Figure 2 for three different collision energies $\left(\sqrt{s_{N N}}\right)$. In order to separate the trivial kinematic broadening of the $d N_{c h} / d \eta$ distribution from the more interesting dynamics, the data for $\mathrm{Au}+\mathrm{Au}$ collisions at different energies can be viewed in the rest frame of one of the colliding nuclei. Such an approach led to the ansatz of "limiting fragmentation" [8], which successfully predicted the energy dependence of particle production away from mid-rapidity in hadron collisions, including $\mathrm{pA}$ [9] and $\overline{\mathrm{p}}$ [10]. This ansatz states that, at high enough collision energy, both $d^{2} N / d y^{\prime} d p_{T}$ and the mix of particle species (and therefore also $d N / d \eta^{\prime}$ ) reach a limiting value and become independent of energy in a region around $y^{\prime} \sim 0$, where $y^{\prime} \equiv y-y_{\text {beam }}$ and rapidity $y \equiv \tanh ^{-1} \beta_{z}$, with the $\hat{z}$ axis defined as the beam (collision) axis. 
Figure 6 shows the scaled, shifted pseudorapidity distributions $d N_{c h} / d \eta^{\prime} /\left\langle N_{\text {part }} / 2\right\rangle$ [四]. The results are folded about mid-rapidity (positive and negative $\eta$ bins are averaged). The distributions are observed to be independent of collision energy over a substantial $\eta^{\prime}$ range. This is consistent with and extends a similar observation made by BRAHMS [11] over a more restricted $\eta^{\prime}$ range. Both the 19.6 and $130 \mathrm{GeV}$ data reach $85-90 \%$ of their maximum value before deviating significantly (more than 5\%) from the common limiting curve.

The data presented here demonstrate that limiting fragmentation applies in the $\mathrm{Au}+$ $\mathrm{Au}$ system, and that the "fragmentation region" in $\mathrm{Au}+\mathrm{Au}$ is rather broad, covering more than half of the available range of $\eta^{\prime}$ over which particles are produced. In particular, the fragmentation region grows significantly between $19.6 \mathrm{GeV}$ and $130 \mathrm{GeV}$, extending more than two units away from the beam rapidity. Particle production appears to approach a fixed limiting curve which extends far from the original beam rapidity, indicating that this limiting curve is an important feature of the overall interaction and not simply a nuclear breakup effect. This result is in sharp contrast to the boost-invariance scenario [12] which predicts a fixed fragmentation region and a broad central rapidity plateau that grows in extent with increasing energy.

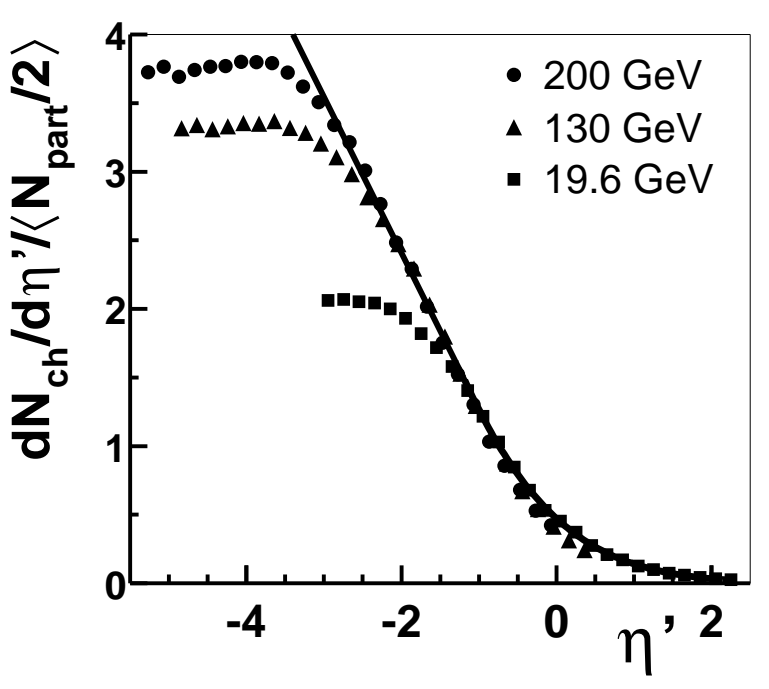

Figure 6: Shifted pseudorapidity distribution, $d N_{c h} / d \eta^{\prime}$ per participant pair, where $\eta^{\prime} \equiv \eta-y_{\text {beam }}$, for $\mathrm{Au}+\mathrm{Au}$ data at $\sqrt{s_{N N}}=$ 19.6, 130, and $200 \mathrm{GeV}$. Systematic errors not shown. The curve is to "guide the eye".

\section{SIMILARITY OF AA AND $e^{+} e^{-}$AT HIGH ENERGY}

In the upper part of Figure 7 , total charged multiplicity data from pp, $\bar{p}, \mathrm{e}^{+} \mathrm{e}^{-}$, and AA (scaled by $\left\langle N_{\text {part }}\right\rangle / 2$ ) are shown as a function of the appropriate $\sqrt{s}$ for each system. See Ref. [13 for more details and [14 for the non-PHOBOS data. The $\mathrm{e}^{+} \mathrm{e}^{-}$data serves as a reference sample, describing the behavior of a simple color dipole system with a large $\sqrt{s}$. The curve is a description of the $\mathrm{e}^{+} \mathrm{e}^{-}$data, given by the functional form: $C \alpha_{s}(s)^{A} e^{\sqrt{B / \alpha_{s}(s)}}$ with the parameters A and B calculable in perturbative QCD and the constant parameter $\mathrm{C}$ determined by a fit to the $\mathrm{e}^{+} \mathrm{e}^{-}$data [15]. In order to compare them with $\mathrm{e}^{+} \mathrm{e}^{-}$, the $\mathrm{pp}$ and $\mathrm{p} \overline{\mathrm{p}}$ data were plotted at an effective energy $\sqrt{s_{\text {eff }}}=\sqrt{s} / 2$, which approximately accounts for the "leading particle effect" [16]. Finally, central AA collisions, $\mathrm{Au}+\mathrm{Au}$ from the AGS and RHIC, and $\mathrm{Pb}+\mathrm{Pb}$ from CERN are shown. Over the available range of RHIC energies from 19.6 to $200 \mathrm{GeV}$, the $\mathrm{Au}+\mathrm{Au}$ results are consistent with the $\mathrm{e}^{+} \mathrm{e}^{-}$results, suggesting a universality of particle production at high energy. In addition, the $\mathrm{Au}+\mathrm{Au}$ data approximately agrees with the scaled pp and $\mathrm{p} \overline{\mathrm{p}}$ data suggesting that the effective energy of a high energy AA collision is approximately just $\sqrt{s_{N N}}$. The lower part of Figure 7 shows these same results, but divided by the $\mathrm{e}^{+} \mathrm{e}^{-}$ fit. This figure illustrates the approach of AA from lower energies towards the high energy 


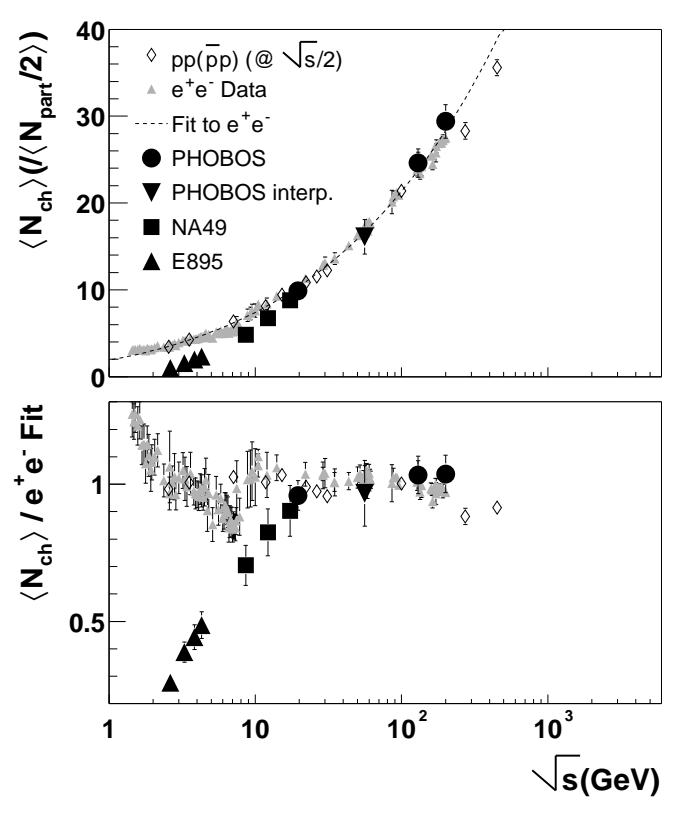

Figure 7. Comparison of the total charged multiplicity versus collision energies for $\mathrm{AA}, \mathrm{e}^{+} \mathrm{e}^{-}$, pp, and $\mathrm{p} \overline{\mathrm{p}}$ data, as described in the text. In the upper panel, the curve is a perturbative QCD expression fit to the $\mathrm{e}^{+} \mathrm{e}^{-}$data. In the lower panel, the data have all been divided by the $\mathrm{e}^{+} \mathrm{e}^{-}$fit.

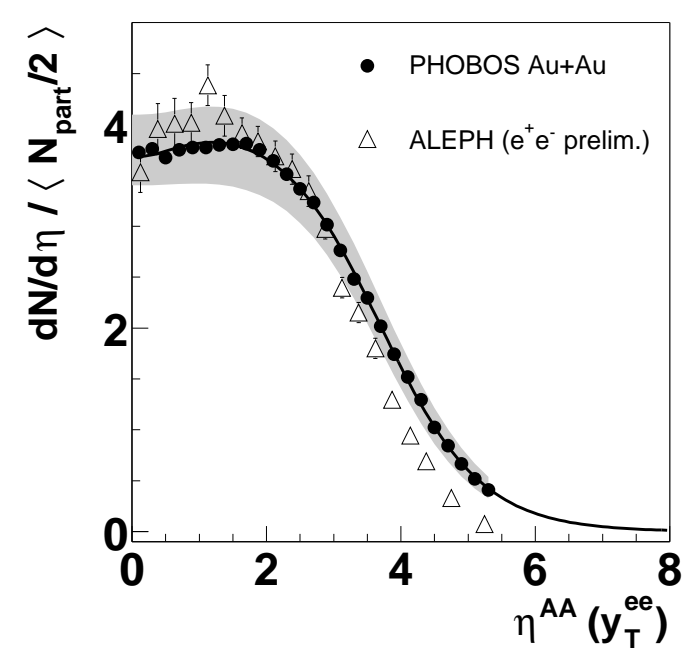

Figure 8. Pseudorapidity distribution of charged particles produced in central $\mathrm{Au}+$ $\mathrm{Au}$ collisions at $\sqrt{s_{N N}}=200 \mathrm{GeV}$ compared with the rapidity distribution along the thrust axis of particles produced in $\mathrm{e}^{+} \mathrm{e}^{-}$collisions at $\sqrt{s}=200 \mathrm{GeV}$. The $\mathrm{Au}+\mathrm{Au}$ data are normalized by $N_{\text {part }} / 2$. Systematic errors are shown for the $\mathrm{Au}+$ Au data.

scaling result.

Figure 8 compares the pseudorapidity distribution for central $\mathrm{Au}+\mathrm{Au}$ collisions to the closest available analog in $\mathrm{e}^{+} \mathrm{e}^{-}$collisions: the thrust-axis-rapidity distribution, where the pion mass was used for all charged particles in $\mathrm{e}^{+} \mathrm{e}^{-}$[17. This shows that particle production in $\mathrm{Au}+\mathrm{Au}$ and $\mathrm{e}^{+} \mathrm{e}^{-}$collisions approximately agree in longitudinal particle distribution as well as in the total yield.

\section{PARTICIPANT SCALING OF PARTICLE PRODUCTION}

Participant scaling was first established in the context of the total charged particle multiplicity produced in pA collisions [18]. In high energy AA collisions, small deviations from participant scaling for mid-rapidity $d N / d \eta$ have been seen. For instance, Figure 9 shows the mid-rapidity $d N / d \eta$ scaled by $\left\langle N_{\text {part }}\right\rangle / 2$ measured with the same apparatus for $\sqrt{s_{N N}}=19.6,130$, and $200 \mathrm{GeV}$. Results of this type are sometimes interpreted in terms of a mixture of "hard" and "soft" components scaling as $N_{\text {part }}^{4 / 3}$ and $N_{\text {part }}$ respectively [19]. This interpretation is somewhat ambiguous, however, since saturation models [19,20 de- 


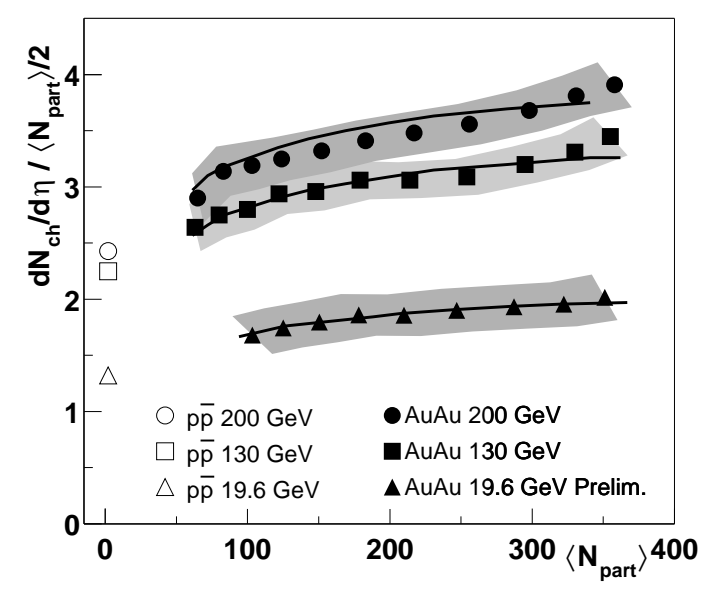

Figure 9. Scaled pseudorapidity density at mid-rapidity, $d N / d \eta /\left\langle N_{\text {part }} / 2\right\rangle$, as a function of centrality for 19.6, 130, and $200 \mathrm{GeV}$. The curves correspond to predictions from the saturation model [19,20].

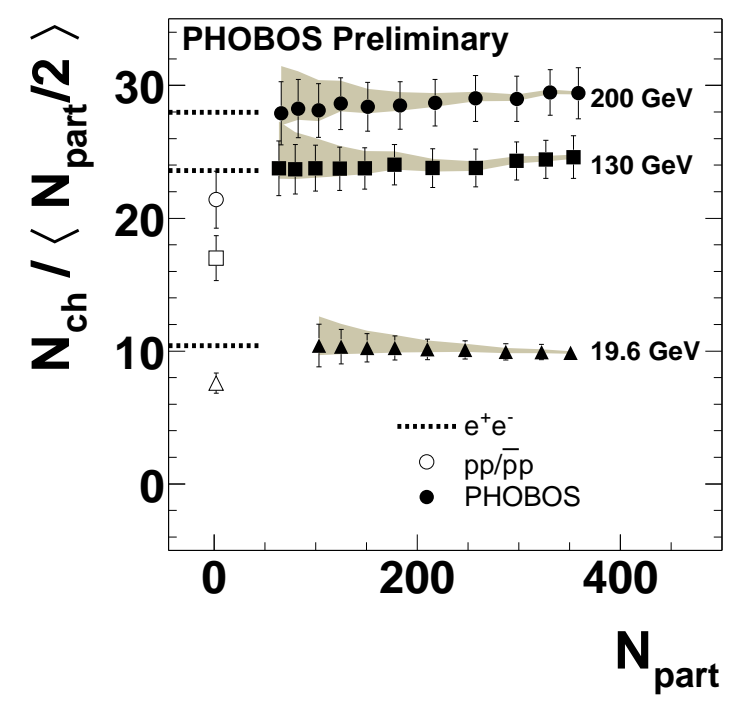

Figure 10. Total charged multiplicity normalized by $\left\langle N_{\text {part }} / 2\right\rangle$ as a function of $N_{\text {part }}$ for AA. Also shown are results for $\mathrm{e}^{+} \mathrm{e}^{-}$ and $\mathrm{p} \overline{\mathrm{p}}$ data.

scribe the data more economically, as can be seen in Figure 9, and since both dynamic and kinematic effects can shift particles around in pseudorapidity as centrality changes.

Due to the large acceptance of the PHOBOS detector, we can integrate the total charged particle production in these collisions. Figure 10 shows $N_{c h} /\left\langle N_{\text {part }} / 2\right\rangle$ as a function of $\left\langle N_{\text {part }}\right\rangle$ for the three different energies. Also shown are the equivalent results for pp and $\mathrm{p} \overline{\mathrm{p}}$ as well as $\mathrm{e}^{+} \mathrm{e}^{-}$collisions at the same values of $\sqrt{s}$. It should be noted that the $\mathrm{pp} / \mathrm{p} \overline{\mathrm{p}}$ data are shown at their collision $\sqrt{s}$ for this plot and not $\sqrt{s_{\text {eff }}}$. Three conclusions can be drawn from this result. First, total charged multiplicity in high energy AA collisions approximately scales with wounded nucleons $\left(N_{\text {part }}\right)$. Second, total particle production in AA collisions per participant pair is the same as the total particle production in $\mathrm{e}^{+} \mathrm{e}^{-}$at the same energy for a broad range of centralities, not just for central collisions. Finally, particle production in $\mathrm{pp}$ and $\mathrm{p} \overline{\mathrm{p}}$ collisions is reduced compared to $\mathrm{AA}$ and $\mathrm{e}^{+} \mathrm{e}^{-}$.

While the overall charged particle production scales with $N_{\text {part }}$, at high $p_{T}$, particle production is expected to scale with $N_{\text {coll }} \propto N_{\text {part }}^{4 / 3}$. Deviations from this scaling at high $p_{T}$ are likely to indicate high density effects in the initial or final state. PHENIX and STAR have already shown that high $p_{T}$ hadron production is suppressed with respect to $N_{\text {coll }} \times \sigma_{p p \rightarrow h X}$ [21]. Recalling that Figure 10 indicates that pp collisions may not be the ideal reference sample, we investigate the scaling of high $p_{T}$ hadron production in AA collisions internally, using a mid-central $\mathrm{Au}+\mathrm{Au}$ collision as a reference rather than pp data.

Figure 11] shows $d^{2} N / d y d p_{T} /\left\langle N_{\text {part }} / 2\right\rangle$ as a function of $\left\langle N_{\text {part }}\right\rangle$ scaled by the value of 
the most peripheral point for $0.5<y<1.3$ for various values of $p_{T}$. The centrality bins used here are specified in Figure 4. The solid curve in the lower right-hand panel is $\left(N_{\text {coll }} / N_{\text {part }}\right) /\left(N_{\text {coll }} / N_{\text {part }}\right)_{\text {periph. }}$. This curve shows the expectation if $N_{\text {coll }}$ scaling for $\mathrm{Au}+\mathrm{Au}$ collisions held true over the centrality range shown. This ratio varies by a factor of two over this centrality range, and yet the particle production approximately scales with $N_{\text {part }}$ for most $p_{T}$ bins. This confirms the empirical observation that particle production at high $p_{T}$ is suppressed with respect to the "hard scaling" expectation. However, we note the additional intriguing fact that, for $N_{\text {part }}>65$, we see approximate $N_{\text {part }}$ scaling of both low and high $p_{T}$ particle production. Mid-range particles with $p_{T} \sim 1.5 \mathrm{GeV} / \mathrm{c}$ show a small violation of $N_{\text {part }}$ scaling, but are still suppressed with respect to the naïve scaling expectations. This particular form of "high $p_{T}$ suppression" could be an indication of initial state suppression (e.g. parton saturation) or that the final state suppression (e.g. "jet quenching") reaches a geometric maximum involving one power of length scale $R_{A u} \propto N_{\text {part }}^{1 / 3}$. Of course, this apparent $N_{\text {part }}$ scaling could also be accidental.

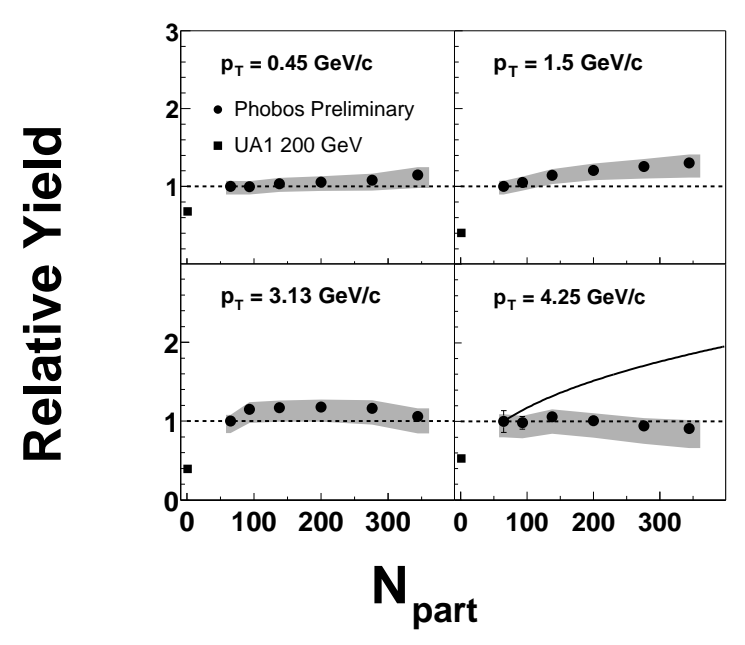

Figure 11: Charged hadron yield in $\mathrm{Au}+\mathrm{Au}$ per participant pair $\left(\left\langle N_{\text {part }}\right\rangle / 2\right)$, normalized to the yield of the most peripheral bin (45$50 \%)$ as a function of $\left\langle N_{\text {part }}\right\rangle$ for different $p_{T}$. The grey bands represent the $90 \%$ C.L. systematic error. The solid line in the lower right-hand panel shows the expectation from $N_{\text {coll }}$ scaling.

\section{SUMMARY}

The PHOBOS experiment has measured a systematic dataset of $\mathrm{Au}+\mathrm{Au}$ collisions at the RHIC collider. These data span an energy range from $\sqrt{s_{N N}}=19.6$ to $200 \mathrm{GeV}$, a pseudorapidity range from -5.4 to +5.4 and a centrality range from 65 to 340 participating nucleons. It also includes particle spectra covering the $p_{T}$ range from 0.03 to $5.0 \mathrm{GeV} / \mathrm{c}$.

Three empirical observations have emerged from this dataset. First, there is clear evidence of limiting fragmentation in $\mathrm{Au}+\mathrm{Au}$ collisions: an energy-independent region of $\eta^{\prime}\left(\eta^{\prime} \equiv \eta-y_{\text {beam }}\right)$. This energy-independent region grows with energy, allowing only a limited region (if any) of longitudinal boost-invariance. Second, there is a striking, unexplained, similarity of particle production in $\mathrm{e}^{+} \mathrm{e}^{-}$and particle production per participating nucleon pair in $\mathrm{Au}+\mathrm{Au}$ collisions. Finally, particle production has been found to approximately scale with number of participating nucleon pairs for $\left\langle N_{\text {part }}\right\rangle>65$. This scaling occurs for the total multiplicity and also for high $p_{T}$ particles (including $\left.3<p_{T}<4.5 \mathrm{GeV} / \mathrm{c}\right)$.

These empirical observations serve, at least, to characterize heavy ion collisions in an economical way and to challenge models. At best, the observed scaling and universalities may point the way to a fairly simple partonic description of this high density matter and 
thus advance our knowledge of the strong interaction.

Acknowledgments: This work was partially supported by U.S. DOE grants DE-AC02-98CH10886, DE-FG02-93ER40802, DE-FC02-94ER40818, DE-FG02-94ER40865, DE-FG02-99ER41099, and W-31-109-ENG-38 as well as NSF grants 9603486, 9722606 and 0072204. The Polish group was partially supported by KBN grant 2-P03B-10323. The NCU group was partially supported by NSC of Taiwan under contract NSC 89-2112-M-008-024.

\section{REFERENCES}

1. R. Nouicer et al., Nucl. Instrum. Methods. A461 (2001) 143, B. B. Back et al., Nucl. Phys. A698 (2002) 416c.

2. B. B. Back et al., Phys. Rev. Lett. 85 (2000) 3100.

3. B. B. Back et al., Phys. Rev. C65 (2002) 061901R.

4. B. B. Back et al., arXiv:nucl-ex/0210015, submitted to Phys. Rev. Lett.

5. B. B. Back et al., arXiv:nucl-ex/0205021, Phys. Rev. Lett. in press.

6. S. Manly et al., proceedings of this conference, arXiv:nucl-ex/0210036.

7. B. Wosiek et al., proceedings of this conference, arXiv:nucl-ex/0210037.

8. J. Benecke, T. T. Chou, C-N. Yang, E. Yen, Phys. Rev. 188 (1969) 2159.

9. J.E. Elias et al., Phys. Rev. D 22 (1980) 13.

10. G. J. Alner et al., Z. Phys. C33, (1986) 1.

11. I. G. Bearden et al., Phys. Rev. Lett. 88, (2002) 202301.

12. J. D. Bjorken, Phys. Rev. D27, (1983) 140.

13. P. A. Steinberg et al., proceedings of this conference, arXiv:nucl-ex/0211002, B. B. Back et al., PRL, to be submitted.

14. J. Klay, U.C. Davis PhD. Thesis (2001), S. V. Afanasiev et al., arXiv:nucl-ex/0205002 (2002), D. E. Groom et al., Eur. Phys. J. C15 (2000) 1.

15. A. H. Mueller, Nucl. Phys. B213 (1983) 85.

16. M. Basile et al., Phys. Lett. B92 (1980) 367, M. Basile et al., Phys. Lett. B95 (1980) 311.

17. P. Abreu et al., Phys. Lett. B459 (1999) 397.

18. J. E. Elias et al., Phys. Rev. Lett. 41 (1978) 285, A. Białas, B. Bleszyński, W. Czyż, Nucl. Phys. B111 (1976) 461.

19. D. Kharzeev, M. Nardi, Phys. Lett. B507 (2001) 121.

20. D. Kharzeev, E. Levin, Phys. Lett. B523 (2001) 79, D. Kharzeev, E. Levin, M. Nardi, arXiv:hep-ph/0111315.

21. K. Adcox et al., Phys. Rev. Lett. 88 (2002) 022301, C. Adler et al., Phys. Rev. Lett. 89 (2002) 202301.

22. C. Roland et al. proceedings of this conference. 